\title{
Model order reduction of linear time invariant systems
}

\author{
Lj. Radić-Weissenfeld ${ }^{1,2}$, S. Ludwig ${ }^{1,2}$, W. Mathis ${ }^{1}$, and W. John ${ }^{2}$ \\ ${ }^{1}$ Leibniz University of Hannover, Institute of Electromagnetic Theory, Hannover, Germany \\ ${ }^{2}$ Advanced System Engineering (ASE), Fraunhofer Institute for Reliability and Microintegration (IZM), Paderborn, Germany
}

\begin{abstract}
This paper addresses issues related to the order reduction of systems with multiple input/output ports. The order reduction is divided up into two steps. The first step is the standard order reduction method based on the multipoint approximation of system matrices by applying Krylov subspace. The second step is based on the rejection of the weak part of a system. To recognise the weak system part, Lyapunov equations are used. Thus, this paper introduces efficient solutions of the Lyapunov equations for port to port subsystems.
\end{abstract}

\section{Introduction}

The order reduction of a linear time invariant system is applied in almost all fields of electrical engineering. The use of order reduced models for test simulations of complex systems is a lot easier than utilising full order models. This is due to the fact that the lower order transfer function can be analysed more easily. Therefore, order reduction algorithms are standard techniques in the integrated circuits community for analysis, approximation and simulation of models arising from interconnect and electromagnetic structure analysis.

The combination of order reduction algorithms, derived from a Krylov subspace projection, and singular value based methods are successfully used for the order reduction of passive linear electrical networks. The Krylov subspace projection method is commonly used for models with a low number of input/output ports. The singular value based methods are successfully used for models of a modest order, i.e. a few hundred. The singular value based method is used for models which matrix representation allows the inversion operation. The classical approach is to use the Krylov subspace

Correspondence to: Lj. Radić-
Weissenfeld
(radic@tet.uni-hannover.de)

projection to more easily obtain the balanced model for singular value based methods of an order reduction.

In this work, these two order reduction methods are combined in two steps. The first step of order reduction algorithm is based on the use of the Krylov subspace projection for the selection of dominant moments, which are found through moment matching. In the second step, the order reduced model is further reduced by rejecting its weak states. A standard matrix inversion is avoided in the second step of the order reduction. This combination of order reduction methods keeps low magnitude and phase errors of order reduced systems.

\section{Standard two-step order reduction}

A two-step order reduction method usually consist of the Krylov subspace projection method (Boley, 1994) and the balanced truncation method (Antoulas and Sorensen, 2001). The Krylov subspace projection produces a system of an order which is proportional to the number of input/output ports. Moreover, the order of the reduced system depends on the number of expansion points and matched moments. Therefore for systems with a high number of input/output ports, the order of the order reduced system may be much higher than necessary. The required part of the system can be reduced by employing a Lyapunov balanced based order reduction method.

Models taken from an integrity circuit (IC) analysis as an IC conducted emission model of $p$ input/output ports, can be described by using MNA as an MIMO system

$$
\begin{aligned}
\mathbf{C} s \boldsymbol{X} & =\mathbf{G} \boldsymbol{X}+\mathbf{B} \mathbf{U} \\
\mathbf{V} & =\mathbf{L} \boldsymbol{X},
\end{aligned}
$$

where $\mathbf{U}, \mathbf{V} \in \mathbb{R}^{p \times 1}$ are input and output vectors, while $\mathbf{C}$, $\mathbf{G} \in \mathbb{R}^{N \times N}, \mathbf{B}, \mathbf{L}^{T} \in \mathbb{R}^{N \times p}$ are system matrices of the order 
$N$. After the order reduction by the Krylov subspace projection the order reduced system is described by

$$
\begin{aligned}
\widetilde{\mathbf{C}} s \tilde{\boldsymbol{X}} & =\widetilde{\mathbf{G}} \tilde{\boldsymbol{X}}+\widetilde{\mathbf{B}} \mathbf{U} \\
\mathbf{V} & =\widetilde{\mathbf{L}} \tilde{\boldsymbol{X}},
\end{aligned}
$$

where $\widetilde{\mathbf{C}}, \widetilde{\mathbf{G}} \in \mathbb{R}^{q \times q}, \widetilde{\mathbf{B}}, \widetilde{\mathbf{L}}^{T} \in \mathbb{R}^{q \times p}$ are order reduced system matrices of the order $q \ll N$. Now the Lyapunov equations are created from the order reduced system described in Eq. (2) by inversion of the matrix $\widetilde{\mathbf{C}}$. This is possible as long as the relevant matrix is not singular. Unfortunately this matrix is normally singular, but due to numerical inaccuracy its determinant differs from zero. For the invertible matrix $\widetilde{\mathbf{C}}$ the Lyapunov equations are

$$
\begin{aligned}
\widetilde{\mathbf{C}}^{-1} \widetilde{\mathbf{G}} \mathbf{P}+\mathbf{P}\left(\widetilde{\mathbf{C}}^{-1} \widetilde{\mathbf{G}}\right)^{T} & =-\widetilde{\mathbf{B}} \widetilde{\mathbf{B}}^{T} \\
\mathbf{Q} \widetilde{\mathbf{C}}^{-1} \widetilde{\mathbf{G}}+\left(\widetilde{\mathbf{C}}^{-1} \widetilde{\mathbf{G}}\right)^{T} \mathbf{Q} & =-\widetilde{\mathbf{L}}^{T} \widetilde{\mathbf{L}} .
\end{aligned}
$$

The balancing can take place if above equations have unique solutions, commonly known as observability $\mathbf{P}$ and controllability $\mathbf{Q}$ Grammians.

\section{Proposed two-step order reduction}

The proposed two-step order reduction algorithm consists of the Krylov subspace projection and truncation of the weak part of a Lyapunov balanced system. The difference in respect to the standard approach, described in the previous section, is going to be discussed in the following.

It is well known that the order of a system reduced by applying the Krylov subspace projection strongly depends on the number of its input/output ports. There are published works that deal with this topic, such as Feldmann (2004), Silva et al. (2006) and Ludwig et al. (2008). In Feldmann (2004) a system of a high number of input/output ports is described so that the input/output ports are not directly involved in the order reduction of a system. Authors in Silva et al. (2006) introduce the correlation matrix with respect to which the weak coupled input/output ports are easily recognised by looking at the input signal. For this work, the method introduced in Ludwig et al. (2007) is employed. The proposed method reduces the number of inputs/outputs, with respect to the model functionality. In contrast to Silva et al. (2006) the input and output signals are not needed. Moreover, the proposed method in Ludwig et al. (2007) can be used for systems with bidirectional ports, for which the method described in Feldmann (2004) is not the most suitable. In Ludwig et al. (2007), it is suggested to replace all independent sources that have the same waveform with the same number of controlled sources and only one port.

Controlled sources and the RLCG part of the system can be efficiently reduced by the projection of a system matrices on the Krylov subspace. The system matrices are matrices obtained by MNA. They do not have a full rank, so that their order $N$ exceeds the amount of state space variables. The order reduced system is described by Eq. (2).
In the second-step of the order reduction, either a whole system or a part of a system that describes a port to port relation can be reduced. To achieve an efficient reduction of a system that was reduced in the first-step, it is divided up into port to port subsystems, so that a subsystem between any port $l$ and any port $m$ of the system is described by the following transfer function

$\widetilde{\boldsymbol{H}}_{l m}=\sum_{k=1}^{q} \frac{\widetilde{r}_{l m k}}{s \widetilde{d}_{k}-1}+s \widetilde{H}_{l m \infty}+\widetilde{H}_{l m 0}$.

Poles $1 \widetilde{d}_{k}$ of all port to port transfer functions originate from matrices $\widetilde{\mathbf{C}}$ and $\widetilde{\mathbf{G}}$. These two matrices are equal for all port to port transfer functions, so that all port to port transfer functions have equal poles. Since transfer functions have different $\widetilde{\mathbf{B}}$ and $\widetilde{\mathbf{L}}$ matrices, they have also different residues $\widetilde{r}_{l m k}$. Each port to port transfer function has also different constant $\widetilde{H}_{l m 0}$ and a polynomial function of the degree one $\widetilde{H}_{l m \infty}$, because these two parts of a transfer function originate from the residues.

The rational and a polynomial function of the degree one in the Eq. (4) build a state space of a system. Therefore, only the rational part of the transfer function has an impact on the order of the sate space matrices. Hence, in the second-step of the order reduction only the part of the system that builds a state space is affected by the order reduction. Namely, instead of reducing the system matrices, the state space of the system is reduced in the second-step. The state space matrices are created from the rational part of the Eq. (4) as

$$
\begin{aligned}
& \mathbf{A}=\left[\begin{array}{cccc}
1 / \tilde{d}_{1} & 0 & \cdots & 0 \\
0 & 1 / \tilde{d}_{2} & \cdots & 0 \\
\vdots & \vdots & \ddots & \vdots \\
0 & 0 & \cdots & 1 / \tilde{d}_{q}
\end{array}\right], \quad \mathbf{b}=\left[\begin{array}{c}
1 \\
1 \\
\vdots \\
1
\end{array}\right], \\
& \mathbf{c}_{l m}=\left[\begin{array}{llll}
\tilde{r}_{l m 1} & \tilde{r}_{l m 2} & \cdots & \tilde{r}_{l m q}
\end{array}\right] \text {. }
\end{aligned}
$$

Lyapunov matrix equations are now

$$
\begin{aligned}
\mathbf{A P}+\mathbf{P A}^{T} & =-\mathbf{b b}^{T} \\
\mathbf{Q}_{l m} \mathbf{A}+\mathbf{A}^{T} \mathbf{Q}_{l m} & =-\mathbf{c}_{l m}^{T} \mathbf{c}_{l m} .
\end{aligned}
$$

Since matrices $\mathbf{A}$ and $\mathbf{b}$ are independent from the port to port transfer functions, the controllability Grammian $\mathbf{P}$ has to be calculated only once for all port to port transfer functions. Because the matrix $\mathbf{A}$ is diagonal and $\mathbf{b}$ consists of ones, the controllability Grammian is a symmetric matrix

$$
\mathbf{P}=-\left[\begin{array}{cccc}
\frac{\widetilde{d}_{11}^{2}}{2 d_{11}} & \frac{\widetilde{d}_{11} \tilde{d}_{22}}{\widetilde{d}_{11}+\tilde{d}_{22}} & \cdots & \frac{\widetilde{d}_{11} \widetilde{d}_{q q}}{\widetilde{d}_{11}+\tilde{d}_{q q}} \\
\widetilde{d}_{11} \widetilde{d}_{22} & \frac{\widetilde{d}_{22}^{2}}{2 \tilde{d}_{11}+\tilde{d}_{22}} & \cdots & \frac{\widetilde{d}_{q q}}{2 \tilde{d}_{22}+\tilde{d}_{q q}} \\
\vdots & \vdots & \ddots & \vdots \\
\frac{\widetilde{d}_{11} \widetilde{d}_{q q}}{\widetilde{d}_{11}+d_{q q}} & \frac{\widetilde{d}_{22} \tilde{d}_{q q}}{d_{22}+d_{q q}} & \cdots & \frac{\tilde{d}_{q q}^{2}}{2 d_{q q}}
\end{array}\right] .
$$




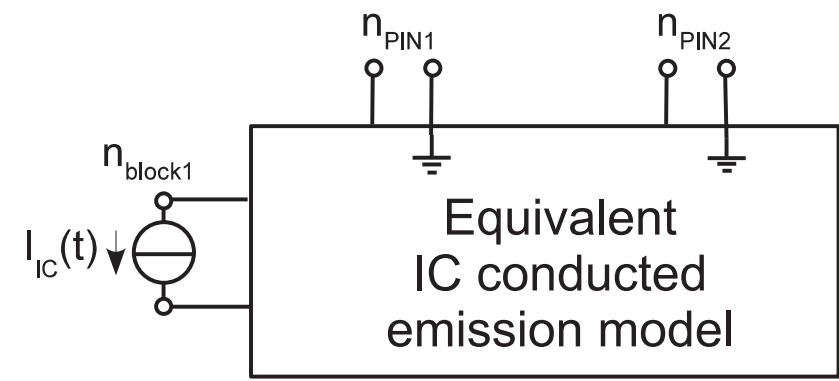

Fig. 1. An IC conducted emission model that consists of two pins, and one added independent current source at the one port, that replaces twelve controlled sources.

Due to the fact that the observability Grammian depends on the residues of the Eq. (4) it differs for each port to port transfer function exposing the symmetry as

$\mathbf{Q}_{l m}=-\left[\begin{array}{ccccc}\widetilde{r}_{l m 1}^{2} \frac{\widetilde{d}_{11}^{2}}{2 \tilde{d}_{11}} & \ldots & \widetilde{r}_{l m 1} \widetilde{r}_{l m q} & \frac{\widetilde{d}_{11} \widetilde{d}_{q q}}{\widetilde{d}_{11}+\tilde{d}_{q q}} \\ \widetilde{r}_{l m 1} \widetilde{r}_{l m 2} \frac{\widetilde{d}_{11} \widetilde{d}_{22}}{\widetilde{d}_{11}+\tilde{d}_{22}} & \ldots & \widetilde{r}_{l m 2} \widetilde{r}_{l m q} & \frac{\widetilde{d}_{q q}}{\widetilde{d}_{22}+\tilde{d}_{q q}} \\ \vdots & \ddots & \vdots \\ \widetilde{r}_{l m 1} \widetilde{r}_{l m q} & \widetilde{d}_{11} \widetilde{d}_{q q} & \ldots & \widetilde{r}_{l m q}^{2} & \frac{\widetilde{d}_{q q}^{2}}{2 d_{d q}}\end{array}\right]$.

The complexity of the proposed solutions of Lyapunov equations is $\mathcal{O}\left(n^{2}\right)$, while the complexity of the standard solution is $\mathcal{O}\left(n^{3}\right)$.

By using the singular value decomposition of the product of controllability and observability Grammians the transformation matrix $\mathbf{T}$ for the Lyapunov balancing can be calculated ( $\mathrm{Li}$ et al., 1999). With the change of the state space variables $\mathbf{X}=\mathbf{T} \widehat{\mathbf{X}}$ in Eq. (1) the balanced system is obtained. Now the Schmidt-Mirsky theorem (Antoulas and Sorensen, 2001) can be used to reduce the state space matrices of the balanced system. The transfer function Eq. (4) after the second-step of order reduction is now

$\widetilde{\widetilde{\boldsymbol{H}}}_{l m}=\widetilde{\mathbf{c}}_{l m}(s \mathbf{I}+\widetilde{\mathbf{A}})^{-1} \widetilde{\mathbf{b}}+s \widetilde{H}_{l m \infty}+\widetilde{H}_{l m 0}$.

where $\tilde{\mathbf{A}} \in \mathbb{R}^{n \times n}$, and $\widetilde{\mathbf{b}}, \widetilde{\mathbf{c}}^{T} \in \mathbb{R}^{n \times 1}$. After second-step of the order reduction $n \ll q \ll N$ holds.

\section{Examples}

The proposed two-step order reduction will be validated by using an IC conducted emission model. The model is an electrical representation of IC behaviour on high frequencies (Steinecke et al., 2006; Gstoettner et al., 2006). The system of the IC conducted emission model is a MIMO, where a certain amount of input/output ports are engaged with current sources. The remaining ports are used for the voltage supply of the chip.

The observed IC conducted emission model consists of two supply pins and twelve current sources. The current

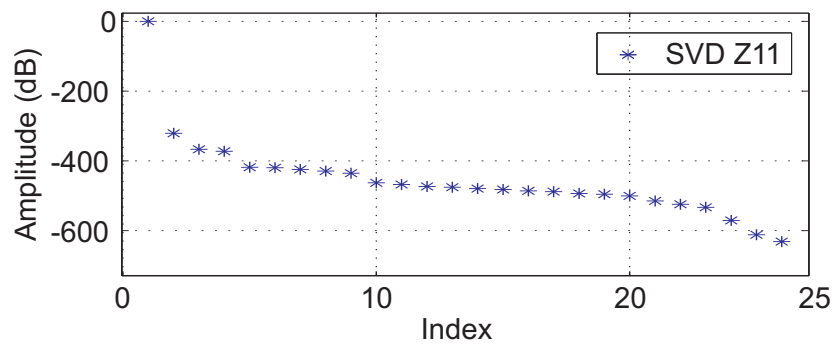

Fig. 2. Hankel singular values of $Z_{11}$ transfer function.

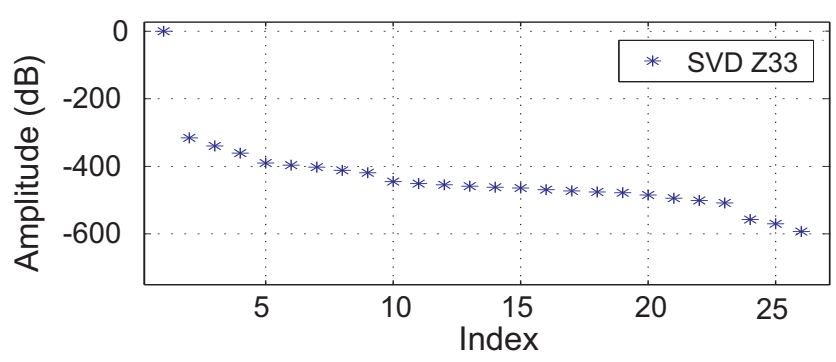

Fig. 3. Hankel singular values of $Z_{33}$ transfer function.

sources are collected into one node, so that the IC conducted emission model has three inputs/output ports. The system is described by six transfer functions in mean of $Z$-parameters. In Fig. 1 ports $n_{\text {PIN1 }}$ and $n_{\text {PIN2 }}$ are supply pins, while port $n_{\text {block1 }}$ is the equivalent current source.

The low order of the system depicted in Fig. 1 can be achieved by using only one expansion point and one moment. However, the transfer function of the order reduced system does not match the original transfer function even in low frequencies.

So, if the order reduction is split into two steps, the order reduced system exhibits more similarities to the original. Namely, in the first-step the original transfer function of the IC conducted emission model is projected on the Krylov subspace, by utilising three expansion points and three moments. The order of the projected system matrices is 27 . Now the transfer function of the reduced system is split into rational and nonrational parts as in Eq. (4). The rational part of the transfer function contains 26 poles. Therefore, the coefficients of the Lyapunov equations are state space matrices of the order 26. The order reduction of the state space matrices depends on the singular values of the system.

Singular values of the transfer functions $Z_{11}$ and $Z_{33}$ are shown in Figs. 2 and 3. The singular values show that the state space matrices that build the transfer functions $Z_{11}$ and $Z_{33}$ can be reduced to the order one. Moreover, the flow of the phase and magnitude of the transfer functions $Z_{11}$ and $Z_{33}$ suggests that the transfer functions can be created by using only one pole, since magnitude and phase have a monotone relation to the frequency. However the order one of the system matrices from Eq. (1) cannot be achieved by using 

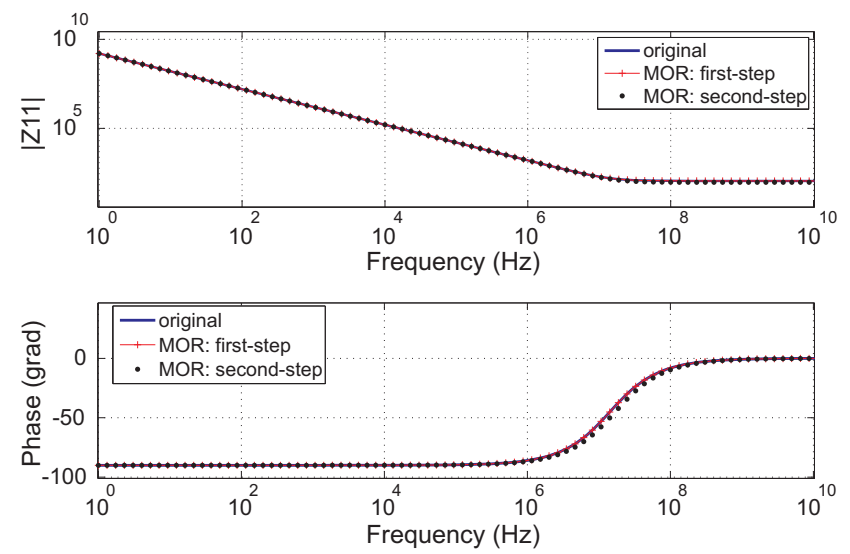

Fig. 4. Magnitude and phase of original (solid line), reduced in firststep (solid plus line) and in second-step (dotted line) $Z_{11}$ transfer function.
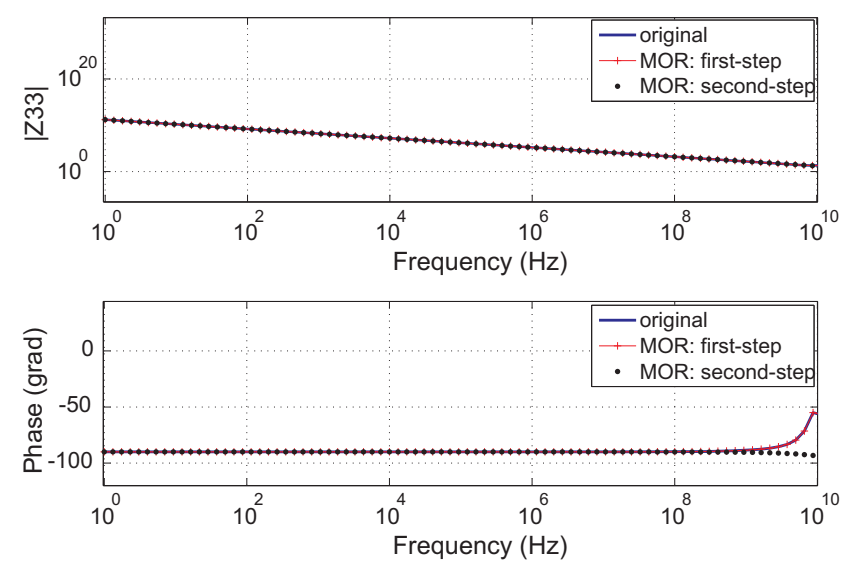

Fig. 5. Magnitude and phase of original (solid line), reduced in firststep (solid plus line) and in second-step (dotted line) $Z_{33}$ transfer function.

only the projection of the system matrices on the Krylov subspace without a loss in system information. By using the suggested two-step order reduction algorithm the system of the IC conducted emission model can be reduced to the order one, while preserving both magnitude or phase characteristics in the whole frequency range of interest.

The results of order reduction are plotted in Figs. 4 and 5. The negligible magnitude and phase differences between original and order reduced transfer functions occur for both transfer functions. The good matching between original and order reduced transfer functions is the consequence of good selection of the expansion points in the first-step and good selection of the transformation matrix in the second-step of the order reduction.

\section{Conclusions}

The first-step of the order reduction is pure projection of each system matrix on the Krylov subspace. For implementation of the second-step of the algorithm, the transfer function is described in mean of residues and poles. This description enables the partitioning of the transfer function onto the rational, constant and as the case may be a polynomial function of the degree one. Since the constant and the polynomial functions are not of importance for a system order, only the rational part of the transfer function is consider in the secondstep of the algorithm. In this way the state space matrices of the system are reduced instead of the system matrices, what enables the creation of very low order system.

The two-step order reduction algorithm is validated by reducing the order of the systems of the IC conducted emission models. The proposed algorithm shows better results than standard order reduction algorithm based on projection of the system matrices on the Krylov subspace.

Acknowledgements. Represented research and development work is carried out in the frame of the MEDEA+ project $2 A 701$ PARACHUTE project (Parasitic Extraction and Optimization for Efficient Microelectronic System Design and Application). This particular research is supported by the $\mathrm{BMBF}$ - Bundesministerium fuer Bildung und Forschung Federal Republic of Germany under grant $01 \mathrm{M} 3169 \mathrm{E}$. The responsibility for this publication is held by authors only.

\section{References}

Anatoulas, A. C. and Sorensen, D. C.: Approximation of large-scale dynamica systems: An overview, I. J. A. M., 11(5), 1093-1121, 2001.

Boley, D. L.: Krylov Space Methods on State-space Control Models, Circ. Syst. Signal Pr., 13(6), 733-758, 1994.

Feldmann, P.: Model Order Reduction of Systems with Large Numbers of Terminals, Proceedings of the Design, Automation and Test in Europe Conference and Exhibition, Paris, France, 2, 944947, 2004.

Gstoettner, A., Steinecke T., and Huemer, M.: Activity Based High Level Modeling of Dynamic Switching Currents in Digital IC Modules, EMC Zurich 2006, Singapore, 2006.

Li, J.-R., Wang, F. and White, J.: An Efficient Lyapunov EquationBased Approach for Generating Reduced-Order Models of Interconnect, Proceedings of 36th Design Automation Conference, New Orleans, LA, USA, 1-6, 1999.

Ludwig, S., Radić-Weissenfeld, Lj., Mathis, W., and John, W.: Model Order Reduction of Integrated Circuit Conducted Emission Models, Proceedings of the EMC Compo, Torino, Italy, November 2007, 215-219, 2007.

Ludwig, S., Radić-Weissenfeld, Lj., Mathis, W., and John, W.: Efficient Network Description of IC Conducted Emission Models for Model Reduction, Adv. Radio Sci., in press, 2008.

Silva, J. M., Vilena, J. F., Flores, P., and Miguel, L.: Outstanding Issues in Model Order Reduction, Proceedings of the Scientific Computing in Electrical Engineering, Sinaia, Romania, September 2006, 11, 139-152, 2006.

Steinecke, T., Goekcen, M., and Gstoettner, A.: High-Accuracy Eission Simulation Models for VLSI Chips including Package and Printed Circuit Board, EMC Compo 2006, Torino, Italy, 41-46, 2006. 